\title{
The Use of Growth Factors and Mesenchymal Stem Cells in Orthopaedics
}

\author{
Medha Kanitkar ${ }^{1}$, Hiteshkumar D. Tailor ${ }^{*}, 1$ and Wasim S. Khan ${ }^{2}$ \\ ${ }^{1}$ University College London Medical School, Gower Street, London WC1E 6BT, UK \\ ${ }^{2}$ University College London Institute of Orthopaedics and Musculoskeletal Sciences, Royal National Orthopaedic \\ Hospital, Stanmore, Middlesex, HA7 4LP, UK
}

\begin{abstract}
Stem cell therapy is an exciting and upcoming branch of tissue engineering with application in the field of orthopaedics. The most commonly used type of stem cells, mesenchymal stem cells (MSCs), can be easily isolated from bone marrow or synovium and cultured in vitro. Newer techniques using tissue engineering to regenerate musculoskeletal tissue by using biomimetic materials are now being studied. These osteoconductive three dimensional constructs seeded with MSCs are highly porous, biodegradable and biomechanically stable scaffolds which do not evoke an immunogenic host cell response. Research has shown the importance of growth factors in guiding and modulating the differentiation of MSCs in order to obtain the required cell type. Gene-based delivery systems have aided the delivery of sustained quantities of these growth factors. The evidence from growth factor enhanced tissue engineering studies for tissue healing looks very positive. This is a multi-disciplinary approach that integrates molecular, biochemical and clinical techniques with developmental and engineering processes. Initial studies indicate an immense potential for cell based strategies to enhance current orthopaedic approaches in skeletal tissue reconstruction. Ultimately, there is a need for randomised controlled trials on human populations to apply these findings to a clinical setting. Nevertheless, stem cell based tissue engineering in orthopaedics shows a promising future.
\end{abstract}

Keywords: Biomaterial, bone, cartilage, growth factors, mesenchymal stem cells, regeneration, tissue engineering.

Much of the interventions in orthopaedics today aim towards the restoration of normal function by replacing damaged tissues or organs in such a way so as to achieve effective, long-lasting and stable repair [1]. A number of traditional therapeutic approaches to repair skeletal tissue have been developed and hypothesised, but they have all presented with a variety of shortcomings. For example, using bone grafts to repair large segmental bone defects [2], extensive bone loss or destruction [3] and avascular necrosis [4] still poses several challenges. Autologous bone graft, which is commonly used, has the disadvantages of limited availability, donor site morbidity, pain, infection and neurovascular injury [5]. The gold standard in bone repair remains sterilised human allogenic bone graft, i.e. tissue donated from other human individuals [6]. However, due to poor osseointegration of allografts, failure rates of up to $30 \%$ have been seen [7].

Another example is articular cartilage damage as a result of trauma or chronic degenerative changes. Cartilage heals very poorly, owing to the lack of intrinsic regenerative capacity of the chondrocytes and its poor vascular supply. In addition, the repaired tissue is more fibrous and mechanically inferior to normal hyaline cartilage resulting in an inadequate physiological repair [8]. Traditional surgical intervention rarely restores the damaged tissue to its original biological state.

\footnotetext{
*Address correspondence to this author at the University College London Medical School, Gower Street, London WC1E 6BT, UK; Tel: +44 207679 0858; Fax: 0207679 0890;

E-mails:mr.htailor@gmail.com, htailor@doctors.org.uk
}

Amidst this, tissue engineering using stem cells has emerged as a hot topic of research and is a promising alternative to the traditional techniques of bone grafting. This method aims to regenerate rather than repair skeletal tissue defects. A stem cell is an immature or undifferentiated cell which is capable of producing identical daughter cells of specific cell lineages [9, 10]. Stem cells have numerous advantages over mature cells for tissue regeneration. Mature cells lack the capacity to extensively self-renew or proliferate in order to generate sufficient yield. They are also limited to a particular phenotype and are thus, usually unable to transdifferentiate into the desired cell lineage upon stimulation.

\section{SOURCES OF STEM CELLS IN SKELETAL TISSUE REGENERATION}

Mesenchymal stem cells are multipotent and have ability to self-renew and proliferate over generations without significant loss of their characteristics [11]. They do not present with the ethical and legal concerns associated with embryonic stem cells and unlike committed chondrocytes isolated from hyaline cartilage, they retain their transdifferentiation capability. MSCs were first described in the $1960 \mathrm{~s}$ by Friendenstein et al. [12]. They demonstrated that rare clonogenic cells isolated from adult rodent bone marrow (BM) aspirates [13] rapidly proliferated in vitro giving rise to distinct colonies, i.e. fibroblastic colonyforming units (CFU-F). These colonies, derived from a single precursor cell, can differentiate into all the different cell lineages of the limb mesoderm (osteoblasts, chodrocytes, adipocytes etc.) [14]. This property gives the potential to generate virtually billions of ex vivo-expanded MSCs from relatively small samples of BM aspirates. 
Tissue engineering is a multidisciplinary area of research. It aims to regenerate tissue by the implantation of cells grown ex vivo, or by stimulating the cells to grow into an implanted matrix [15]. The ex vivo expansion of these cells into all types of mesenchymal tissue is paramount to the success of cell-based tissue engineering. MSC-based therapy can be used for tissue regeneration by both site-specific (localised) and systemic applications.

Its basic principle involves the use of living cells with a natural or synthetic support, or scaffold, to produce a threedimensional (3D) living tissue construct that is implantable and can facilitate a faster rate of tissue repair or fill recipient tissue site. To engineer the new tissue, donor cells are first isolated, harvested and seeded onto the scaffold. The next step is the stimulation of cell proliferation and then the maintenance of cellular differentiation. Finally, the living tissue is implanted into the patient in order to obtain the $3 \mathrm{D}$ construct containing the newly seeded living cells in vivo. MSCs are a useful cell source in tissue engineering due to their innate capacity for regeneration and their ability to be easily isolated and expanded using culture techniques.

An ideal tissue engineering scaffold should have some key properties such as being osteoconductive if filling bone site. It should be fully biodegradable, porous, biomechanically stable and non-immunogenic to the host site [16]. Natural biomaterials facilitate better cell attachment, differentiation and function, however, they may vary from batch-to-batch and have difficulties in scaling up by ex vivo expansion of the MSCs. Synthetic biomaterials, on the other hand (of which polyactides are the most widely used), can be engineered according to precise specifications but may not always interact with cells in the desired way. Hence, a biomimetic material which can combine the advantages of both natural and synthetic biomaterials is an ideal scaffold for cell-based tissue engineering.

In a study by Yoon et al. [17], a fully absorbable osteogenic poly (DL-lactide-co-glycolide) (PLGA) scaffold seeded with MSCs was used to repair intercalated bone defects in vivo. Biomaterials can be rendered osteoinductive in several ways and, in this case, the scaffold was loaded with calcitriol, which is shown to induce mineralisation of the bone tissue [18]. The appropriate level of calcitriol in the pores is crucial to support proliferation and differentiation of the cells into osteoblasts. Calcitriol-loaded PLGA scaffolds seeded with/without MSCs were implanted in an animal model of a bone defect. Radiograph assessments showed that only the calcitriol-loaded groups showed bony union at 9 weeks. Also, RT-PCR and histological studies confirmed the development of new osteoid matrix and direct calcium deposits contributing to successful regeneration of the bony defects. At 20 weeks, complete bony union was achieved. The MSCs not only enhanced osteoconduction of the implanted scaffold via angiogenic activation of the host cells, but also underwent differentiation. The PLGA scaffold also degrades completely in 8 weeks without leaving residual metabolites due to its high porosity [19].

However, whether the MSCs themselves differentiated into osteoblasts directly or they promoted angiogenesis of the scaffold remains uncertain. Nevertheless, this fully absorbable synthetic bone graft biomaterial along with marrow stem cells does provide a hopeful allogenic graft substitute to repair large bone defects.

Hydroxyapatite (HA) has been used extensively in orthopaedics, particularly with promoting osteoconduction of prosthesis. Maracci et al. [3] investigated the use of HAcoated ceramic scaffolds seeded with MSCs to repair long bone defects. The MSCs were expanded in vitro and seeded onto the scaffolds. These were placed in the long bone defects of four patients. The patients were followed up over 6-7 years and evaluated using radiographs at fixed intervals. The results showed integration of implants with host bone, new bone formation inside bioceramic pores and vascular ingrowth. However these outcomes were all progressive. Seven months after implant surgery, complete fusion and full functional recovery of the limbs was achieved. This was less than half the recovery time compared to the traditional bone allograft.

Future strategies in this field would be to use more ideal costructs like PLGA which provide the initial support but is resorbed at a similar rate to new bone deposition in the scaffold. It should also provide more stable fixation and sitespecific formation of new bone as shown in animal studies using porous calcium phosphate-based scaffolds [20, 21]. Ultimately, there is a need for randomised controlled trials after performing more animal studies to apply this to a clinical setting. In addition, the cost benefit ratio will also need to be considered after sufficient trials have been carried out to validate the use of this technology over traditional methods of bone reconstruction [2].

\section{GROWTH FACTORS IN ORTHOPAEDICS}

Bone volume is determined by the relative rates of bone formation and resorption. Growth factors are protein signalling agents that act locally to stimulate formation and proliferation of osteoblasts [22]. The multilineage potential of MSCs occurs under guided differentiation to obtain the required cell type. This can only be achieved under the modulation of appropriate growth factors [23]. Once damaged, chondrocytes, especially from articular cartilage have only a limited capacity in self-repair [24]. The use of growth factors extends to regeneration of muscle, tendon, ligament and connective tissue. Hence, growth factors are used to retain MSCs' proliferative capacity and differentiation potential, which is lost during their ex vivo expansion. However, delivering sufficient quantities of growth factor locally in a sustained manner has proven difficult until the advent of gene transfer technology. Combining this gene-based delivery system with tissue bioengineering is a potential approach to achieving more biologically active product at the site of the defect than that delivered by exogenous recombinant proteins [25].

There are a number of growth factors used for regenerative applications in orthopaedics. Some of these will be discussed here.

\section{Bone Morphogenetic Protein (BMP)}

The autograft contains not just the stem cells and the 3D scaffold, but also osteoinductive growth factors, of which human Bone Morphogenetic Protein (BMP) is now frequently used since it was first described by Urist about 40 years ago [26]. BMPs mostly influence bone, but also 
haematopoietic cell differentiation [27]. Gene transfer provides a good method to deliver the BMP signal in a sustained manner [28]. In a study by Evans et al, grafts of muscle or fat were transduced with BMP-2 using an adenovirus vector [29]. The gene-activated fat and muscle were able to heal significantly sized osteochondral defects in rabbit models. Both these tissues possess an intrinsic capacity to regenerate into bone and other connective tissue because they possess osteoprogenitor cells and are able to serve as scaffolds. The healing occurs by an endochondral process [30]. Histological evidence showed a rapid and efficient conversion of muscle into cartilage [29]. The transplanted graft induces the formation of osteoblasts from host osteoprogenitor cells, as also demonstrated by Gerstenfeld et al. [31].

BMPs have been used in fracture non-unions. This is exemplified by the use of both BMP-7 and BMP-2 in long bone fracture non-unions [32-34]. Clinical studies looking at long bone non-union fractures treated with BMP, bone healing rates were between $75-100 \%$ and the mean healing times were all under 8 months [32, 33, 35-37]. The nonunion healing with conventional gold-standard treatment of autograft [34] reaches similar levels of $87-100 \%$, however as mentioned earlier they also carry several risks such as donor graft morbidity [5].

\section{Transforming Growth Factor- $\beta 1$ (TGF-- $\beta 1$ )}

TGF- $\beta$ is a growth factor which helps stimulate cell replication and extracellular matrix formation [38]. Autologous chondrocytes obtained from MSCs can undergo expansion and proliferation in the presence of a chondrogenic medium containing TGF- $\beta 1$ to the $3 \mathrm{D}$ culture. MSCs have the capacity to synthesise all components of the normal articular cartilage matrix [39].

A study by Guo et al. [40] investigated whether TGF- $\beta 1$ modified MSCs to enhance the repair of full thickness articular cartilage damage in a rabbit model. TGF- $\beta 1$ is not only chondrogenic, but also has the ability to inhibit the alloreactive immune response. The MSCs transfected with TGF- $\beta 1$ gene were seeded onto a biomimetic scaffold (polyL-lysine coated polylactide (PLA)) in vitro and allografted into the cartilage defects. Macroscopic, histological and ultrasound studies were performed at specific follow-up times. The in vitro study showed that the proliferation and generation of cartilaginous matrix by the experimental group was substantially greater than in the control group, MSCs without TGF- $\beta 1$. Furthermore, the animal model studies provided evidence of surface hyaline cartilage-specific extracellular matrix synthesis and reconstruction of the subchondral bone at 24 weeks post-implantation. An important criterion of gene based tissue engineering is that growth factor delivery should have a sustained expression, as demonstrated [40].

Another study [41] looked at chondrogenesis of MSCs in vitro using TGF- $\beta 1$ enhanced biphasic implants which were made up of a gel and a solid scaffold. The gel component in these implants ensures homogenous distribution of the cells. In addition, human and animal fibrin has proven useful in the storage and delivery of many growth factors [42]. The study showed that the biphasic implants embedded with locally releasing bioactive TGF- $\beta 1$ showed enhanced chondrogenic proliferation in vitro compared to the classical pellet culture.

Park et al. described an enhanced neocartilage formation by rabbit MSCs using a hydrogel plus heparin-bound TGF- $\beta$ growth factor [43]. The results indicated a long-term release of TGF- $\beta$ after complexation with chondroitin sulfate suitable for induction of chondrogenic differentiation of rabbit MSCs [44].

The use of TGF- $\beta$ to augment the effect of the MSCs on the scaffold has thus, shown to enhance the recruitment, proliferation and differentiation of the bone marrow stem cells [45].

TGF- $\beta 1$ has also been employed in inducing arthrofibrosis in a study by Watson et al. [46] and its potential as a pro-fibrotic in joint disease. The study investigated how sustained production of TGF- $\beta 1$ intraarticularly drives chronic arthrofibrosis. A recombinant adenovirus gene vector to locally overexpress TGF- $\beta 1$ in a rat model and this induced a fibrotic condition that immobilised the injected joint. In 10 days, the proliferation of fibroblasts replaced all the normal anatomical structures and began to fuse with the articular cartilage, ligamentous and capsular fibrous tissue. Also, the levels of collagen I, III and $\mathrm{V}$ were elevated by 10 -fold. At 30 days, the cartilaginous masses had penetrated the entire joint space and deposited a rich proteoglycan matrix and matrix metalloproteinases (MMPs) were shown to promote tissue remodelling on histological examination.

\section{Vascular Endothelial Growth Factor (VEGF)}

Bone formation and angiogenesis are closely linked. Therapeutic angiogenesis is a new concept that promotes new blood vessel formation by delivering exogenous angiogenic growth factors to the tissue such as VEGF [47]. Before effective osteogenesis occurs, there needs to be the formation of new blood vessels that invade and mediate the supply of oxygen, nutrients and osteoprogenitor cells [48].

Both TGF- $\beta 1$ and VEGF were locally administered using transfected bone marrow stem cells to repair the anterior cruciate ligament using the Achilles tendon in a study by Wei et al. [49]. The VEGF significantly improved revascularization of the graft but TGF- $\beta 1$ was needed to aid mechanical strength by regulating the formation and crosslinkage of collagen type I and type III. The best mechanical properties of the graft were found to be at 24 weeks. Hence, the co-expression of these two genes was found to produce the best results.

In a study by Geiger et al. [50] large bone defects were treated with VEGF transfected bone marrow stem cells using a coralline scaffold (bone substitue). Four different groups were analysed. In the control, the scaffold was coated with a control-plasmid DNA. In group 2 it was coated with VEGF plasmid DNA, group 3 with MSC-transfected control plasmid and finally group 4 with both MSCs and the VEGF plasmid. The results showed that both the VEGF-transfected cell groups had increased vascularisation and osteogenesis. In addition, the VEGF plus MSC group led to more intense and homogenous vascularisation of the defect and fastest resorption of the bone substitute. 


\section{Basic Fibroblast Growth Factor (bFGF)}

It has been reported in two studies that the transfection of TGF- $\beta 1$ gene with MSCs can also induce them to express bFGF and VEGF [22, 51]. These can promote the regeneration of vasculature, thereby enhancing the osteogenic differentiation of MSCs. This was also supported by another study by Guo et al. [2], where bFGF transfected MSCs seeded on biomimetic biodegradable scaffolds were used to repair large segmental bone defects. Both the in vitro and in vivo evidence pointed to significantly more bone formation and abundant active angiogenesis in the experimental groups.

In another study [52], different FGFs were added to a chondrogenic medium at different stages of development. This chondrogenesis in a pellet culture using transfected MSCs occurred by three stages as in embryonic skeletal development. These were: condensation, differentiation and hypertrophy which were clearly expressed as seen by the different levels of expression of collagen II and N-cadherin. During condensation, the cells expressed cell adhesion molecules which become downregulated as they differentiated to chondrocytes, forming a cartilage template. Next, the cartilage hypertrophied and finally, ossified. FGF receptor activation is associated with endochondral ossification during development. These receptors were initially discovered when it was found that their mutations caused abnormalities in skeletal development [53]. In the future, modulating the FGF signal to produce stage-specific differentiation in chondrogenesis remains to be explored. A better knowledge of as well as control over the FGF receptors will lead to further advancements in cartilage healing and extend to regeneration of other types of tissue.

We have previously shown that synovial fat pad derived MSCs expanded in fibroblast growth factor-2 (FGF-2) showed cell surface epitope expression similar to adult stem cells [54]. The synovial fat pad derived MSCs showed chondrogenic differentiation in cell aggregate cultures, and prior expansion with FGF-2 enhanced chondrogenesis.

Thus, the evidence from growth factor enhanced tissue engineering studies points to a great potential for bone, cartilage, tendon and muscle healing.

\section{CONCLUSIONS}

Mesenchymal stem cells, that are the most commonly used type of cells can be easily isolated and cultured in vitro. Harnessing these osteoprogenitor cells that have the ability to differentiate into all the different cell lineages of the limb mesoderm gives them the potential to generate virtually billions of ex vivo-expanded MSCs that can be implanted at the site of the defect, hence a relatively non-invasive technique. However, being able to isolate a demonstrably pure, multipotent and self-renewing stem cell sub fraction from bone marrow stroma, or in fact any other tissue source continues. Future research should aim to focus on purification of these stem cells in addition to trying to fully understand the potential of these primitive cells so they can be harnessed to their full potential to enhance tissue repair.

Osteoconductive 3D constructs seeded with MSCs is a highly porous, biodegradable and biomechanically stable scaffold which does not evoke an immunogenic host cell response. These bio-engineered graft substitutes make an ideal alternative to the traditional bone/cartilage grafts, showing no donor site morbidity and being completely resorbed from the host after a period of time. Future strategies would involve designing a scaffold which provides stable fixation and maximised strength along with sitespecific formation of new skeletal tissue.

Growth factors contribute angiogenic and regenerative potential along with mechanical stability in the differentiated tissue. Harnessing this capacity of growth factors as extracellular signalling agents can be used for the differentiation of bone and other tissues to mimic the natural development process [55]. Gene-based delivery systems have aided the delivery of sustained quantities of these growth factors but further exploration of associated risks and benefits as a source for cell-based tissue regeneration in longitudinal animal studies is required.

Ultimately, there is a need for randomised controlled trials on human populations to apply these findings to a clinical setting. In addition, the cost benefit ratio will also need to be considered after sufficient trials have been carried out to validate the use of this technology over traditional methods of tissue reconstruction.

\section{ACKNOWLEDGEMENT}

None declared.

\section{CONFLICT OF INTEREST}

None declared.

\section{REFERENCES}

[1] Vacanti, JP, Langer R. Tissue engineering and design and fabrication of living replacement devices for surgical reconstruction and transplantation. Lancet 1999; 354(Suppl 1): 132-4.

[2] Guo X, Zheng Q, Kulbatski I, et al. Bone regeneration with active angiogenesis by basic fibroblast growth factor gene transfected mesenchymal stem cells seeded on porous $\beta$-TCP ceramic scaffolds. Biomed Mater 2006; 1: 93-9.

[3] Marcacci M, Kon E, Moukhachev V, et al. Stem cells associated with macroporous bioceramics for long bone repair: 6- to 7-year outcome of a pilot clinical study. Tissue Eng 2007; 13: 947-55.

[4] Kim YM, Lee SH, Lee FY, et al. Morphologic and biomechanical study of avascular necrosis of the femoral head. J Orthop 1991; 14: 1111-6.

[5] Arrington ED, Smith WJ, Chambers HG, et al. Complications of iliac crest bone graft harvesting. Clin Orthop 1996; 329: 300-9.

[6] Slooff TJ, Buma P, Schreurs BW, et al. Acetabular and femoral reconstruction with impacted graft and cement. Clin Orthop Relat Res 1996; 324: 108-15.

[7] Sorger JI, Hornicek FJ, Zavatta M, et al. Allograft fractures revisited. Clin Orthop Relat Res 2001; 382: 66-74.

[8] Furukawa T, Eyre DR, Koide S, Glimcher MJ. Biochemical studies on repair cartilage resurfacing experimental defects in the rabbit knee. J Bone Joint Surg Am 1980; 62: 79-89.

[9] Watt FM, Hogan BL. Out of Eden: stem cells and their niches. Science 2000; 287: 1427-30.

[10] Robey PG. Stem cells near the century mark. J Clin Invest 2000; 105: 1489-91.

[11] Cancedda R, Mastrogiacomo M, Bianchi G et al. Bone marrow stromal cells and their use in regenerating bone. Novartis Found Symp 2003; 249: 133-43.

[12] Friedenstein AJ, Shapiro-Piatetzky II, Petrakova KV. Osteogenesis in transplants of bone marrow cells. J Embryol Exp Morphol 1966; 16: 381-90.

[13] Friedenstein AJ, Deriglasova UF, Kulagina NN, et al. Precursors for fibroblasts in different populations of hematopoietic cells as detected by the in vitro colony assay method. Exp Hematol 1974; 2: 83-92. 
[14] Bianco P, Riminucci S, Gronthos P, et al. Bone marrow stromal stem cells: nature, biology, and potential applications. Stem Cells 2001; 19: 180-92.

[15] Stock UA, Vacanti JP. Tissue engineering: current state and prospects. Annu Rev Med 2001; 52: 443-51

[16] Masahiro Y, Hidetomi T, Yuuki I. Repair of intercalated long bone defect with a synthetic biodegradable bone-inducing implant. Biomaterials 2005; 26: 5145-52.

[17] Yoon S J, Park KS, Kim MS. Repair of diaphyseal bone defects with calcitriol-loaded PLGA scaffolds and marrow stromal cells. Tissue Eng 2007; 13: 1125-33.

[18] Lee S J, Lee D H, Khang G, et al. Preparation and characterization of $1,25(\mathrm{OH}) 2$ vitamin D3 loaded the biodegradable PLGA scaffolds for tissue engineered bone. Macromol Chem Symp 2002; 15: 201 .

[19] Tatebe M, Nakamura R, Kagami H, et al. Differentiation of transplanted mesenchymal stem cells in a large osteochondral defect in rabbit. Cytotherapy 2005; 7: 520-30.

[20] Kon E, Muraglia A, Corsi A, et al. Autologous bone marrow stromal cells loaded onto porous hydroxyapatite ceramic accelerate bone repair in critical-size defects of sheep long bones. J Biomed Mater Res 2000; 49: 328-37.

[21] Mastrogiacomo M, Corsi A, Francioso E, et al. Reconstruction of extensive long bone defects in sheep using resorbable bioceramics based on silicon stabilized tricalcium phosphate. Tissue Eng 2006; 12: 1261-73.

[22] Mohan S, Baylink DJ. Bone growth factors. Clin Orthop Relat Res 1991; 263: 30-48.

[23] Pittenger MF, Mackay AM, Beck SC, et al. Multilineage potential of adult human mesenchymal stem cells. Science 1999; 284: 143-7.

[24] Buckwalter JA. Articular cartilage: injuries and potential for healing. J Orthop Sports Phys Ther 1998; 28: 192-202.

[25] Franceschi RT. Biological approaches to bone regeneration by gene therapy. J Dent Res 2005; 84: 1093-103.

[26] Urist MR, Iwata H, Ceccotti PL, et al. Bone morphogenesis in implants of insoluble bone gelatin. Proc Natl Acad Sci USA 1973; 70: 3511-5.

[27] Chadwick K, Wang L, Li L, et al. Cytokines and BMP-4 promote hematopoietic differentiation of human embryonic stem cells. Blood 2003; 102: 906-15.

[28] Evans CH, Ghivizzani SC, Robbins PD. Orthopaedic gene therapy. Clin Orthop Relat Res 2004; 429: 316-29.

[29] Evans CH, Liu FJ, Glatt V, et al. Use of genetically modified muscle and fat grafts to repair defects in bone and cartilage. Eur Cell Mater 2009; 18: 96-111.

[30] Ketenjian AY, Jafri AM, Arsenis C. Studies on the mechanism of callus cartilage differentiation and calcification during fracture healing. Orthop Clin North Am 1978; 9(1): 43-65.

[31] Gerstenfeld LC, Cruceta J, Shea CM, et al. Chondrocytes provide morphogenic signals that selectively induce osteogenic differentiation of mesenchymal stem cells. J Bone Miner Res 2002; 17: 221-30.

[32] Kanakaris NK, Lasanianos N, Calori GM, et al. Application of bone morphogenetic proteins to femoral non-unions: a 4-year multicentre experience. Injury 2009; 40 (Supp1 3): S54-61.

[33] Calori GM, D'Avino M, Tagliabue L. An ongoing research for evaluation of treatment with BMPs or AGFs in long bone nonunion: protocol description and preliminary results. Injury 2006; 37(Suppl 3): S43-S50.

[34] Crowley DJ, Kanakaris NK, Giannoudis PV. Femoral diaphyseal aseptic nonunions: is there an ideal method of treatment? Injury 2007; 38 (Suppl 2): S55-63.

[35] Dimitriou R, Dahabreh Z, Katsoulis E. Application of recombinant BMP-7 on persistent upper and lower limb non-unions. Injury 2005; 36(Supp1 4): S51-9.
[36] Johnson EE, Urist MR, Finerma GA. Resistant nonunions and partial or complete segmental defects of long bones. Treatment with implants of a composite of human bone morphogenetic protein (BMP) and autolyzed, antigen-extracted, allogeneic (AAA) bone. Clin Orthop 1992; 277: 229-37.

[37] Ronga M, Baldo F, Zappala G, et al. Recombinant human bone morphogenetic protein-7 for treatment of long bone non-union: an observational, retrospective, non-randomized study of 105 patients. Injury 2006; 37 (Suppl 3): S51-6.

[38] Rifkin DB. Latent transforming growth factor- $\beta$ (TGF- $\beta$ ) binding proteins: orchestrators of TGF- $\beta$ availability. J Biol Chem 2005; 280: 7409-12.

[39] Barry F, Boynton RE, Liu B, et al. Chondrogenic differentiation of mesenchymal stem cells from bone marrow: differentiationdependent gene expression of matrix components. Exp Cell Res 2001; 268: 189-200.

[40] Johnstone B, Hering TM, Caplan AI, et al. In vitro chondrogenesis of bone marrow-derived mesenchymal progenitor cells. Exp Cell Res 1998; 238: 265-72.

[41] Dickhut A, Dexheimer V, Martin K, et al. Chondrogenesis of Human Mesenchymal Stem Cells by Local Transforming Growth Factor-Beta Delivery in a Biphasic Resorbable Carrier. Tissue Engineering Part A 2010; 16: 453-64.

[42] Petratos PB, Felsen D, Trierweiler G, et al. Transforming growth factor-beta2 (TGF-beta2) reverses the inhibitory effects of fibrin sealant on cutaneous wound repair in the pig. Wound Repair Regen 2002; 10: 252-8.

[43] Park JS, Woo DG, Yang HN, et al. Heparin-bound transforming growth factor-beta3 enhances neocartilage formation by rabbit mesenchymal stem cells. Transplantation 2008; 85: 589-96.

[44] Park JS, Yang HJ, Woo DG, et al. Chondrogenic differentiation of mesenchymal stem cells embedded in a scaffold by long-term release of TGF-beta3 complexed with chondroitin sulfate. J Biomed Mater Res A 2010; 92: 806-16.

[45] Huang, Q, Goh JC, Hutmacher DW, Lee EH. In vivo mesenchymal cell recruitment by a scaffold loaded with transforming growth factor betal and the potential for in situ chondrogenesis. Tissue Eng 2002; 8: 469-82.

[46] Watson R, Gouze E, Levings P, et al. Gene delivery of TGF- $\beta 1$ induces arthrofibrosis and chondrometaplasia of synovium in vivo. Lab Invest 2010; 90: 1615-27.

[47] D’Amore PA. Angiogenesis. Sci Med 1999; 6: 44-53.

[48] Schor AM, Canfield AE, Sutton AB, et al. Pericyte differentiation. Clin Orthop 1995; 313: 81-91.

[49] Wei X, Mao Z, Hou Y, et al. Local administration of TGFß$1 /$ VEGF gene-transduced bone mesenchymal stem cells for Achilles allograft replacement of the anterior cruciate ligament in rabbits. Biochem Biophys Res Commun 2011; 406: 204-10.

[50] Geiger F, Lorenz $\mathrm{H}, \mathrm{Xu} \mathrm{W}$, et al. VEGF producing bone marrow stromal cells (BMSC) enhance vascularization and resorption of a natural coral bone substitute. Bone 2007 Volume 41; 4: 516-22.

[51] Evans C, et al. Using gene therapy to protect and restore cartilage. Clin Orthop 2000; 379: S214-9.

[52] Hellingman C, Koevoet W, Kops N, et al. Fibroblast growth factor receptors in in vitro and in vivo chondrogenesis: relating tissue engineering using adult mesenchymal stem cells to embryonic development. Tissue Eng Part A 2010; 16: 545-56.

[53] Ornitz DM. FGF signaling in the developing endochondral skeleton. Cytokine Growth Factor Rev 2005; 16: 205-13.

[54] Khan WS, Tew SR, Adesida AB, Hardingham TE. Human infrapatellar fat pad-derived stem cells express the pericyte marker $3 \mathrm{G} 5$ and show enhanced chondrogenesis after expansion in fibroblast growth factor-2. Arthritis Res Ther 2008; 10(4): R74.

[55] Xiao C, Zhou H, Liu G, et al. Bone marrow stromal cells with a combined expression of BMP-2 and VEGF-165 enhanced bone regeneration. Biomed Mater 2011; 6: 015013. 